\title{
In-Frame Variants in STAG3 Gene Cause Premature Ovarian Insufficiency
}

\begin{abstract}
Wen-Juan Xiao ${ }^{1+}$, Wen-Bin He ${ }^{1,2,3 t}$, Ya-Xin Zhang ${ }^{1}$, Lan-Lan Meng ${ }^{2,3}$, Guang-Xiu Lu ${ }^{1,2,3}$, Ge Lin ${ }^{1,2,3}$, Yue-Qiu Tan ${ }^{1,2,3 * \pm}$ and Juan Du ${ }^{1,2,3 * \neq}$

${ }^{1}$ Institute of Reproduction and Stem Cell Engineering, Central South University, Changsha, China, ${ }^{2}$ Genetic Center, Reproductive and Genetic Hospital of CITIC-Xiangya, Changsha, China, ${ }^{3}$ NHC Key Laboratory of Human Stem Cell and Reproductive Engineering, Central South University, Changsha, China
\end{abstract}

\section{OPEN ACCESS}

Edited by:

Fan Jin,

Zhejiang University, China

Reviewed by:

Ye Wang,

Sun Yat-sen University, China

Xu Chenming,

Shanghai Jiao Tong University,

China

*Correspondence:

Yue-Qiu Tan

tanyueqiu@csu.edu.cn

Juan Du

tandujuan@csu.edu.cn

tThese authors have contributed equally to this work and share first authorship

FThese authors have contributed equally to this work and share senior authorship

Specialty section: This article was submitted to Genetic Disorders,

a section of the journal

Frontiers in Genetics

Received: 17 April 2019 Accepted: 24 September 2019 Published: 14 November 2019

Citation:

Xiao W-J, He W-B, Zhang $Y-X$, Meng L-L, LU G-X, Lin G, Tan Y-Q and Du J (2019) In-Frame Variants in STAG3 Gene Cause Premature

Ovarian Insufficiency.

Front. Genet. 10:1016.

doi: 10.3389/fgene.2019.01016
Premature ovarian insufficiency (POI) is a severe clinical syndrome defined by ovarian dysfunction in women less than 40 years old who generally manifest with infertility, menstrual disturbance, elevated gonadotrophins, and low estradiol levels. STAG3 is considered a genetic aetiology of POI, which facilitates entry of REC8 into the nucleus of a cell and plays an essential role in gametogenesis. At present, only six truncated variants associated with POI have been reported; there have been no reports of an in-frame variant of STAG3 causing POI. In this study, two novel homozygous in-frame variants (c.877_885del, p.293_295del; c.891_893dupTGA, p.297_298insAsp) in STAG3 were identified in two sisters with $\mathrm{POI}$ from a five-generation consanguineous Han Chinese family. To evaluate the effects of these two variants, we performed fluorescence localization and co-immunoprecipitation analyses using in vitro cell model. The two variants were shown to be pathogenic, as neither STAG3 nor REC8 entered nuclei, and interactions between mutant STAG3 and REC8 or SMC1A were absent. To the best of our knowledge, this is the first report on in-frame variants of STAG3 that cause POI. This finding extends the spectrum of variants in STAG3 and sheds new light on the genetic origins of POI.

Keywords: premature ovarian insufficiency, STAG3 gene, in-frame variant, fluorescence localization, co-immunoprecipitation

\section{INTRODUCTION}

Premature ovarian insufficiency (POI), also known as premature ovarian failure, refers to hypergonadotropic hypogonadism in women younger than 40 years and is one of the major causes of female infertility, affecting at least 1-3\% of adult women in the world (Bannatyne et al., 1990). Apart from menstrual disturbance (amenorrhea or oligomenorrhea for at least 4 months), the main symptoms of POI are reduced levels of estradiol and elevated plasma levels of follicle stimulating hormone (FSH) (>25 mIU/ml on two occasions, > 4 weeks apart) (Shelling, 2010; Gowri et al., 2015; Tucker et al., 2016; Webber et al., 2016). The etiology of POI is complex, such as that for autoimmune diseases, chemotherapy, and pelvic surgery, among which genetic defect was reported to be related to POI (Franca et al., 2019).

POI is a condition with extremely high genetic heterogeneity. A set of genes has been reported to be responsible for POI, including X-linked genes (e.g., BMP15 and FMR1) and autosomal genes 
(e.g., HFM1, FIGLA, FOXL2, STAG3, and FSHR) (Rossetti et al., 2017). Stromal antigen 3 (STAG3) is a meiosis-specific gene that is restrictedly expressed in testes and ovaries in humans, and it plays an important role in gametogenesis and fertility (Houmard et al., 2009; Nogues et al., 2009; Garciacruz et al., 2010; Caburet et al., 2014). Variants of STAG3 are rare, and only six truncated variants, three frameshift variants, two nonsense variants, and one splicing variant (Caburet et al., 2014; Stabej et al., 2016; Colombo et al., 2017; He et al., 2018a; Franca et al., 2019) (Table 1) associated with POI have been reported to date. However, it remains enigmatic whether in-frame variants of STAG3 can cause POI and female infertility.

In this study, we identified two novel homozygous in-frame variants of STAG3 in a consanguineous Han Chinese family with POI by whole-exome sequencing (WES). Moreover, we performed in silico and in vitro functional analyses to reveal the relationship between the two variants and POI. To the best of our knowledge, this is the first report of in-frame variants of STAG3 associated with POI, and the first example of performing in vitro functional analyses to functionally characterize in-frame variants of STAG3.

\section{MATERIALS AND METHODS}

\section{Patients and Blood Sampling}

Four members of a five-generation consanguineous Han Chinese family with POI, namely, two affected sisters (V-1; V-2, Figure 1A) and two unaffected second-cousin parents (IV-1; IV-2, Figure 1A) participated in this study. Peripheral blood samples from the four individuals were collected, and genomic DNA (gDNA) was extracted using a QIAamp DNA Blood Midi Kit (Qiagen, Hilden,
Germany). The four family members provided their written informed consent to participate in this study and agreed with the publication of this case report. This study was reviewed and approved by the ethics committee of the Reproductive \& Genetic Hospital of CITIC-Xiangya of Central South University, China.

\section{WES, Variant Filtering, and Sanger Sequencing}

The gDNA from the proband was subjected to WES following protocols described previously (He et al., 2018b). Whole exomes were captured using Agilent SureSelect version 4 (Agilent Technologies, Santa Clara, CA) and sequenced on a HighSeq2000 sequencing platform (Illumina, San Diego, California, USA). WES data analysis was performed using the Genome Analysis Toolkit (GATK) and consisted of removing adaptors, mapping WES raw reads to a reference human genome sequence (NCBI GRCh37, reference genome Hg19) using the Burrows-Wheeler Aligner (BWA), eliminating PCR duplicates, and sorting sequences using Picard (http://broadinstitute.github.io/picard/). Variant identification was performed using the GATK package according to the recommended best practices, including base recalibration variant calling with Haplotype Caller and variant quality score recalibration and annotation using ANNOVAR software.

All candidate variants needed to meet the following criteria: (i) absent or occurring at a frequencyless than 0.01 in any of the following databases: 1,000 Genomes Variant Database, Exome Aggregation Consortium (ExAC), or NHLBI-GO Exome Sequencing Project (GO-ESP); (ii) homozygous variants were considered with priority; (iii) predicted to be deleterious; and (iv) relevant to the phenotype

TABLE 1 | The currently reported phenotypes and genotypes of STAG3 gene in POI patients with 46,XX.

\begin{tabular}{|c|c|c|c|c|c|c|c|c|}
\hline \multirow[t]{2}{*}{ Patients } & \multirow[t]{2}{*}{ Genotype } & \multicolumn{6}{|c|}{ Phenotype } & \multirow[t]{2}{*}{ References } \\
\hline & & $\begin{array}{l}\text { Menstrual } \\
\text { history }\end{array}$ & $\begin{array}{c}\text { FSH } \\
(\mathrm{mlU} / \mathrm{ml})\end{array}$ & $\begin{array}{c}\text { LH } \\
(\mathrm{mlU} / \mathrm{ml})\end{array}$ & $\begin{array}{l}\text { Estradiol } \\
(\mathrm{Pg} / \mathrm{ml})\end{array}$ & $\begin{array}{l}\text { Ultrasonographic } \\
\text { examination }\end{array}$ & $\begin{array}{l}\text { Age at } \\
\text { diagnosis } \\
\text { (years) }\end{array}$ & \\
\hline 1 & $\begin{array}{l}\text { c.877_885del (p.293_295del) } \\
\text { (Hom) c.891_893dupTGA } \\
\text { (p.297_298insAsp) (Hom) }\end{array}$ & $\begin{array}{l}\text { Primary } \\
\text { amenorrhea }\end{array}$ & 51 & 11.08 & $<10$ & $\begin{array}{l}\text { Uterus and ovaries } \\
\text { not visualized }\end{array}$ & 18 & This study \\
\hline 2 & $\begin{array}{l}\text { c.877_885del (p.293_295del) } \\
\text { (Hom)c.891_893dupTGA } \\
\text { (p.297_298insAsp) (Hom) }\end{array}$ & $\begin{array}{l}\text { Primary } \\
\text { amenorrhea }\end{array}$ & 72.19 & 18.58 & $<10$ & $\begin{array}{l}\text { Small uterus and } \\
\text { small ovaries }\end{array}$ & 21 & This study \\
\hline 3 & $\begin{array}{l}\text { c.291dupC (p.Asn98GInfs`2) } \\
\text { (Het)c.1950C > A (p.Tyr650*) } \\
\text { (Het) }\end{array}$ & $\begin{array}{l}\text { Primary } \\
\text { amenorrhea }\end{array}$ & 89 & 37 & $<13$ & $\begin{array}{l}\text { Infantile uterus; ovaries } \\
\text { were not visualized }\end{array}$ & 21 & $\begin{array}{l}\text { (Franca et al., } \\
\text { 2019) }\end{array}$ \\
\hline 4 & c.677C > G (p.Ser227) (Hom) & $\begin{array}{l}\text { Primary } \\
\text { amenorrhea }\end{array}$ & 48 & 23 & 19 & NA & NA & $\begin{array}{l}\text { (Colombo et al., } \\
\text { 2017) }\end{array}$ \\
\hline 5 & $\begin{array}{l}\text { c. } 1573+5 G>A \\
\text { (p.Leu490Thrfs*10) (Hom) }\end{array}$ & $\begin{array}{l}\text { Primary } \\
\text { amenorrhea }\end{array}$ & 48.69 & 26 & normal & $\begin{array}{l}\text { Streak gonads and } \\
\text { small uterus }\end{array}$ & 19 & (He et al., 2018a) \\
\hline 6 & $\begin{array}{l}\text { c. } 1573+5 G>A \\
\text { (p.Leu490Thrfs*10) (Hom) }\end{array}$ & $\begin{array}{l}\text { Primary } \\
\text { amenorrhea }\end{array}$ & 48.38 & 25.51 & normal & NA & NA & \\
\hline 7 & $\begin{array}{l}\text { c.1947_48dupCT } \\
\text { (p.Tyr650Serfs²2) (Hom) }\end{array}$ & $\begin{array}{l}\text { Primary } \\
\text { amenorrhea }\end{array}$ & 136 & 31 & $<37$ & $\begin{array}{l}\text { Normal uterus; bilateral } \\
\text { streak gonads }\end{array}$ & NA & $\begin{array}{l}\text { (Stabej et al., } \\
\text { 2016) }\end{array}$ \\
\hline 8 & $\begin{array}{l}\text { c.1947_48dupCT } \\
\text { (p.Tyr650Serfs²2) (Hom) }\end{array}$ & $\begin{array}{l}\text { Primary } \\
\text { amenorrhea }\end{array}$ & 130 & 62 & $<37$ & $\begin{array}{l}\text { Small uterus; streak } \\
\text { gonads }\end{array}$ & NA & \\
\hline $9-12$ & $\begin{array}{l}\text { c.968delC (p.Gln188Argfs*8) } \\
\text { (Hom) }\end{array}$ & $\begin{array}{l}\text { Primary } \\
\text { amenorrhea }\end{array}$ & $>45$ & $>18$ & $<22$ & $\begin{array}{l}\text { Bilateral streak } \\
\text { gonads }\end{array}$ & $\begin{array}{c}17-20 \\
\text { (4 sisters) }\end{array}$ & $\begin{array}{l}\text { (Caburet et al., } \\
\text { 2014) }\end{array}$ \\
\hline
\end{tabular}

POI, premature ovarian insufficiency; FSH, follicle stimulating hormone; LH, luteinizing hormone; Hom, homozygote variant; Het, heterozygote variant; NA, data not available. 


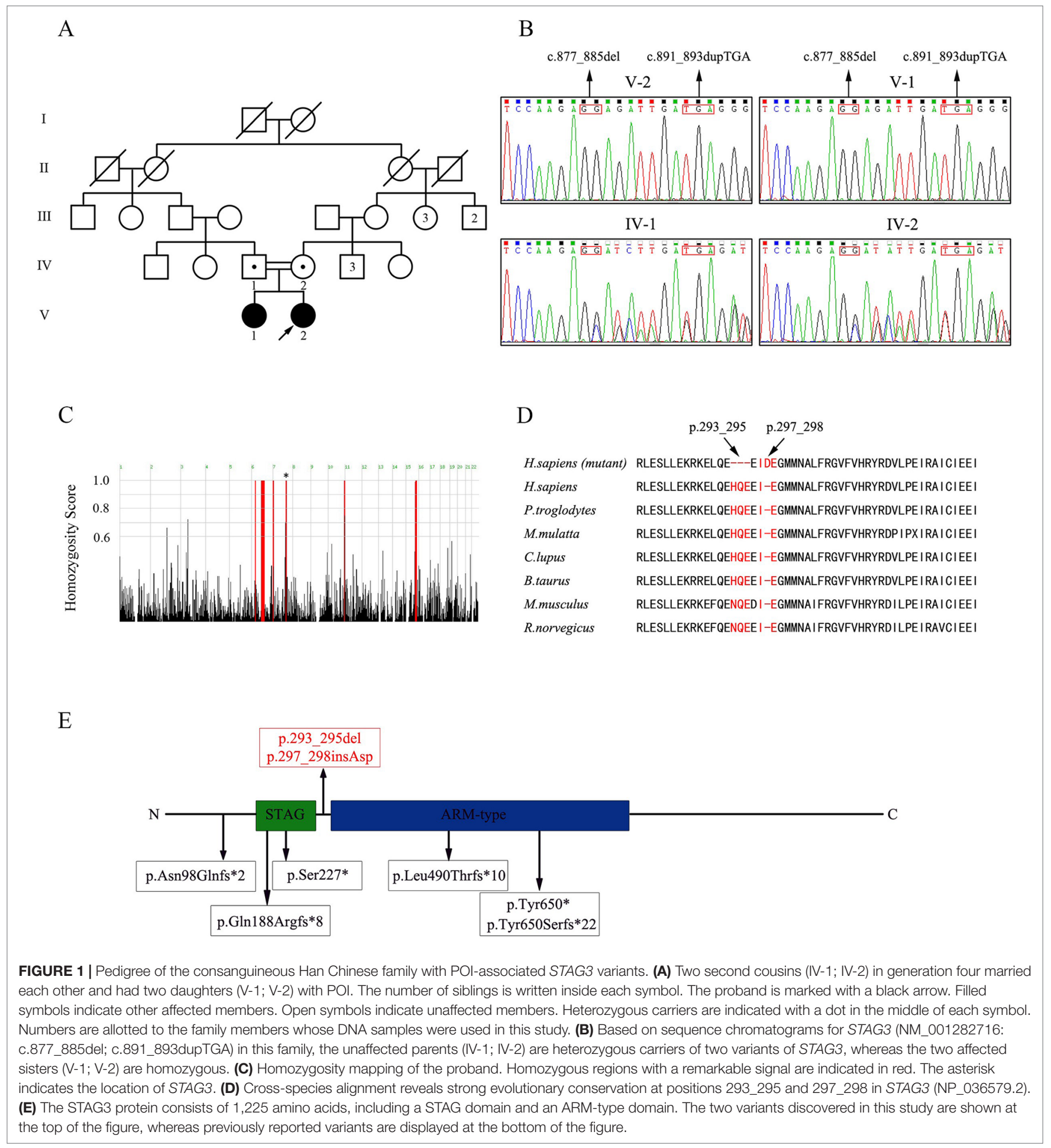

shared by the patients based on comprehensive expression data (expression in ovary), and model organism data (a female-lackingoocytes phenotype presented in animal models).

Specific PCR primers targeting two variants of the STAG3 gene (NM_001282716) were designed and validated. The sequences were as follows: $5^{\prime}$-AACCACATGCAGAGGGGTTAT-3' and 5' -TCCAGCTGCATTAATTCTGGGA-3.'

\section{Plasmid Construction}

The full-length coding sequence of STAG3 was amplified from control human blood cDNA, and the STAG3 product was cloned into pDsRed2-N1, resulting in STAG3 followed by DsRed2 protein, as STAG3-WT-DsRed2. Additionally, the cDNA encoding $\mathrm{FLAG}_{3}$-STAG3 was cloned into pcDNA3.1, namely, FLAG 3 -STAG3-WT. Variants c.877_885del and 
c.891_893dupTGA were separately or simultaneously introduced into the STAG3-WT-DsRed2 and FLAG $3^{-}$ STAG3-WT plasmid vectors using a Mut Express II Fast Mutagenesis Kit V2 (Vazyme, Guangzhou, China) to achieve site-directed mutagenesis. Altogether, six mutants were generated (Mut 1 indicates STAG3-p.293_295delDsRed2 or FLAG 3 -STAG3-p.293_295del; Mut 2 indicates STAG3-p.297_298insAsp-DsRed2 or FLAG 3 -STAG3-p.297_ 298insAsp; Mut 3 indicates STAG3-p.293_295del and p.297_298insAsp-DsRed2 or FLAG 3 -STAG3-p.293_295del and p.297_298insAsp). Moreover, cDNAs encoding REC8 and SMC1A were separately cloned into pEGFP-N1 and pcDNA3.1-HA. All expression constructs were sequenced to exclude unintended PCR-generated variants and confirm the presence of the desired variants.

\section{Cell Culture}

Chinese hamster ovary $(\mathrm{CHO})$ cells and human embryonic kidney (HEK) 293 cells were cultured in Dulbecco's modified Eagle medium nutrient mixture F-12 (Ham) (DMEM/F12) (Gibco, Gaithersburg, MD, USA) with $10 \%$ fetal bovine serum (FBS) (Gibco, Gaithersburg, MD, USA) at $37^{\circ} \mathrm{C}$ in a humidified $5 \% \mathrm{CO}_{2}$ incubator.

\section{Fluorescence Localization}

According to the manufacturer's instructions for Lipofectamine 3000 (Thermo Fisher Scientific, Carlsbad, CA, USA), CHO cells were transfected with expression vectors containing wild-type STAG3-DsRed2 and REC8-EGFP plasmids or mutated STAG3DsRed 2 and REC8-EGFP plasmids. CHO cells transfected with empty vector (EV; without STAG3) and REC8-EGFP plasmids were used as negative controls. $\mathrm{CHO}$ cells were fixed for fluorescence localization after culture for 48 h. 4,6-Diamidino-2phenylindole (DAPI) (Beyotime) was used to visualize DNA. All fluorescent images were captured on an orthostatic fluorescence microscope (Olympus, Japan).

\section{Co-Immunoprecipitation (Co-IP)}

Co-IP was performed as described previously (Wolf et al., 2018). Briefly, HEK293 cells were transfected with expression vectors containing wild-type $F_{2 A G_{3}-S T A G 3}$ and REC8-EGFP plasmids (or SMC1A-HA plasmids) or mutated $F L A G_{3^{-}}$ STAG3 and REC8-EGFP plasmids (or SMC1A-HA plasmids), according to the manufacturer's instructions for Lipofectamine 3000 use. Untransfected HEK293 cells were used as a negative control. After incubation for $36 \mathrm{~h}$, the culture medium was supplemented with $0.2 \mu \mathrm{g} / \mathrm{ml}$ nocodazole (Sigma, Louis, MO, USA) for prometaphase arrest of the cells. Total protein was extracted using RIPA lysis buffer (Thermo Fisher Scientific) after $12 \mathrm{~h}$, and then, western blotting was performed to detect the expression of STAG3, REC8, and SMC1A using anti-FLAG, anti-GFP, and anti-HA (Proteintech) antibodies, respectively, at a 1:1,000 dilution. Next, immunoprecipitation was performed using Dynabeads Protein G (Invitrogen) following the manufacturer's instructions.

\section{RESULTS}

\section{Clinical Features of the Affected Individuals}

The proband (V-2, Figure 1A) initially went to the clinic because of primary amenorrhea and was diagnosed with POI at 18 years old. Her breast development was at Tanner stage I, although she had a normal height $(160 \mathrm{~cm})$ and weight $(50 \mathrm{~kg})$. The results of hormonal studies showed that her serum FSH level was high (51 $\mathrm{mIU} / \mathrm{ml}$, normal range $2-22 \mathrm{mIU} / \mathrm{ml}$ ), whereas her serum estradiol level was low $(<10 \mathrm{pg} / \mathrm{ml}$, normal range $30-190 \mathrm{pg} / \mathrm{ml})$, and her anti-Müllerian hormone (AMH) level was too low to be detected. She had a normal level of luteinizing hormone ( $\mathrm{LH}$; $11.08 \mathrm{mIU} / \mathrm{ml}$, normal range $1-19 \mathrm{mIU} / \mathrm{ml}$ ). Her uterus and ovaries were not visualized upon ultrasonographic examination, and she had an abnormal vulva without labia minora. The proband did not accept hormonal replacement therapy (HRT) because of the increased prevalence of invasive breast cancer after HRT (Biscup, 2003).

The proband's affected elder sister (V-1, Figure 1A) showed normal growth and development but presented with POI when she was 21 years old. Similar to the proband, she went to the hospital because of primary amenorrhea. Her serum FSH level was elevated $(72.19 \mathrm{mIU} / \mathrm{ml}$, normal range $2-22 \mathrm{mIU} / \mathrm{ml})$, whereas her serum estradiol level was low $(<10 \mathrm{pg} / \mathrm{ml}$, normal range $30-190 \mathrm{pg} / \mathrm{ml})$, and her $\mathrm{LH}$ level was normal (18.58 $\mathrm{mIU} / \mathrm{ml}$, normal range 1-19 $\mathrm{mIU} / \mathrm{ml})$. Ultrasonographic examination showed that her uterus was anteflexed and small $(23 \times 10 \times 20 \mathrm{~mm})$. Both ovaries were also small: the left ovary was $14 \times 10 \times 11 \mathrm{~mm}$, and the right one was $15 \times 11 \times 13 \mathrm{~mm}$, consistent with the size of streak gonads. After receiving HRT with progesterone for 3 months, she showed normal menses, and her uterus grew $(36 \times 20 \times 35 \mathrm{~mm})$ to a size close to that of a normal adult-sized uterus.

Additionally, both sisters had a normal karyotype (46, XX) and FMR1 CGG repeats, and no associated adrenal or thyroid autoimmune disorders were found.

\section{Identification of Two Homozygous In-Frame Variants of STAG3}

WES of the proband (V-2, Figure 1A) resulted in approximately $139,631,574$ raw reads with a mean 182.73-fold depth in the target regions, revealinghigh-quality sequencing (Supplementary Table 1). For rare inherited diseases, the frequency of possible pathogenic variants should be very low in a healthy population. Therefore, the results were screened against minor allele frequency (MAF) $>1 \%$ in public SNP and indel databases for variants predicted to be deleterious or to result in loss of function. Homozygous variants were preferentially considered candidates because the patients were from a consanguineous family. Finally, we focused on the phenotypic relevance for POI in the proband. Only two homozygous variants of STAG3 (NM_001282716: c.877_885del, p.293_295del; c.891_893dupTGA, p.297_298insAsp) fulfilled these criteria (Supplementary Table 2).

The two homozygous in-frame STAG3 variants were confirmed by Sanger sequencing and were also detected in her affected sister 
(Figure 1B). Their unaffected parents were heterozygous carriers of the two variants (Figure 1B). In addition, homozygosity mapping indicated that the two STAG3 variants were not located in heterozygous regions (Figure 1C) and that positions p.293_295 and p.297_298 are highly conserved across different species (Figure 1D).

According to the American College of Medical Genetics and Genomics (ACMG) standards and guidelines for the interpretation of variations (Richards et al., 2015), the two in-frame variants of STAG3 (c.877_885del, p.293_295del; c.891_893dupTGA, p.297_298insAsp) are both classified as likely pathogenic variants. Therefore, we speculated that both homozygous STAG3 variants were candidates as causes of POI.

\section{Effects of Mutant STAG3 Proteins on REC8 Localization in CHO Cells}

Co-expression of wild-type STAG3 and REC8 is necessary for REC8 to enter nuclei. A fluorescence localization analysis was performed to evaluate the effects of the two variants. As expected, REC8 localized to nuclei, as did the will-type STAG3 protein, when $\mathrm{CHO}$ cells were transfected with expression vectors containing wild-type STAG3 and REC8 plasmids; (Figure 2A). In contrast, REC8 localized exclusively in the cytoplasm
(Figure 2A) when $\mathrm{CHO}$ cells were co-transfected with the REC8 construct and empty vector or mutant STAG3 construct. In the latter scenario, nearly all of the mutant STAG3 proteins were excluded from nuclei (Figure 2A).

\section{Lack of Interactions Between Mutant STAG3 and REC8 or SMC1A}

We then performed a Co-IP analysis to determine the pathogenicity of the two variants. As shown, wild-type STAG3 interacted with both REC8 and SMC1A as previously reported (Prieto et al., 2001; Wolf et al., 2018) (Figure 2B). Additionally, when we directly examined the ability of the three transiently expressed mutant STAG3 proteins (p.293_295del; p.297_298insAsp; p.293_295del, and p.297_298insAsp) to associate with REC8 or SMC1A, the results revealed that there was no interaction between the three mutant STAG3 proteins and REC8 or SMC1A (Figure 2B).

\section{DISCUSSION}

In the present study, two novel homozygous in-frame variants (c.877_885del, p.293_295del; c.891_893dupTGA,

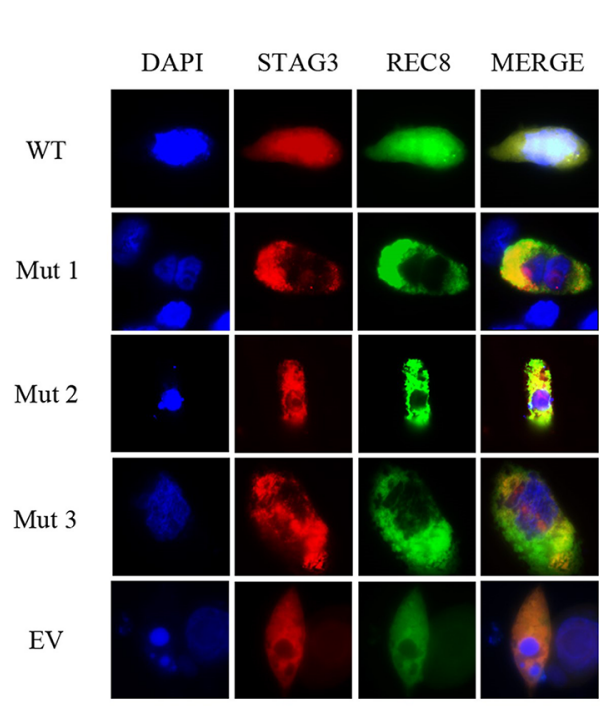

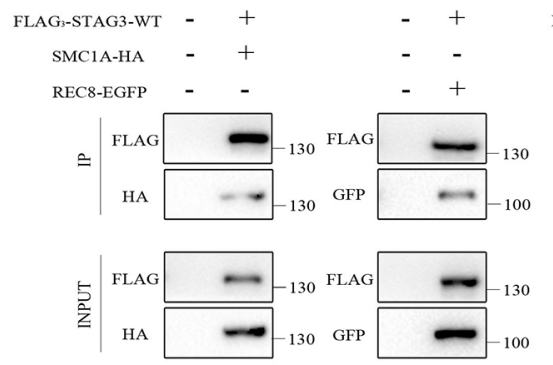
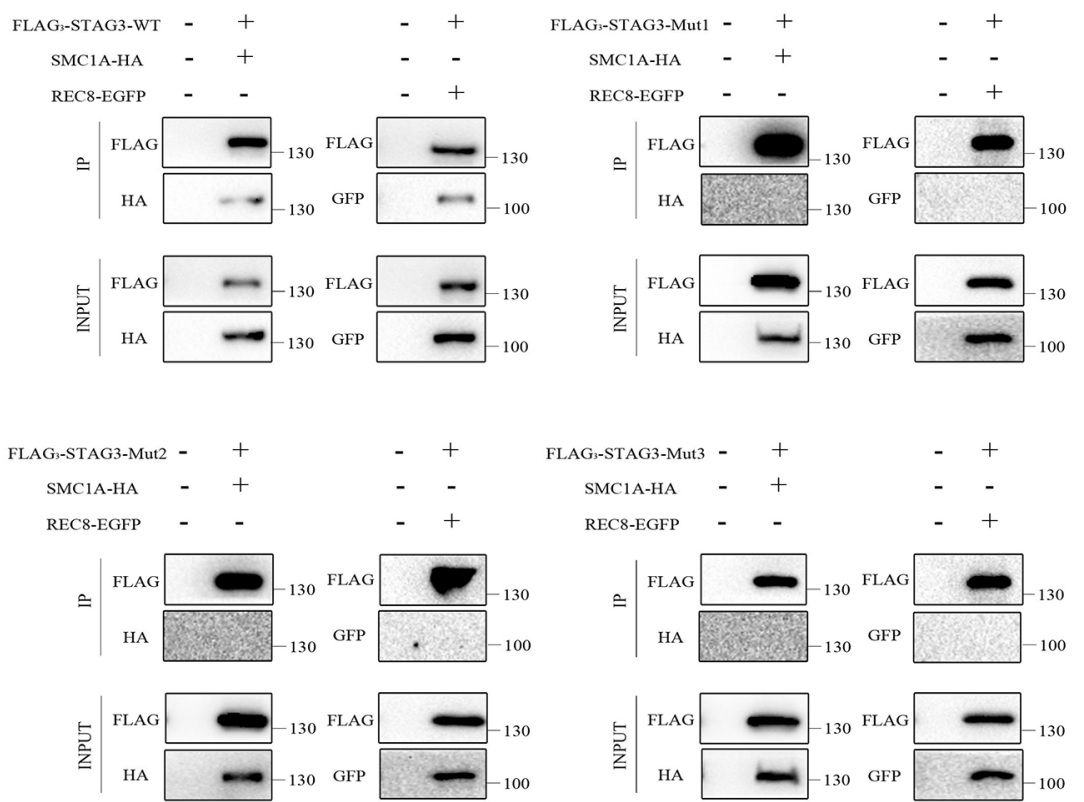

FIGURE 2 | Fluorescence localization and Co-IP analyses of the two in-frame variants of STAG3. (A) CHO cells were transiently transfected with a plasmid encoding STAG3-DsRed2 and REC8-EGFP, mutant STAG3-DsRed2 and REC8-EGFP, or an empty vector and REC8-EGFP. After culture for 48 h, cells were fixed for fluorescence localization. Blue fluorescence indicates DAPI staining in nuclei. The three mutant STAG3 proteins were restricted to the cytoplasm and could not

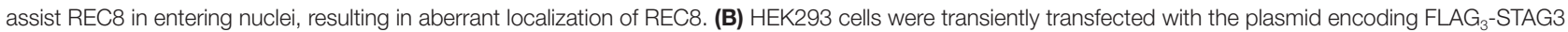
(or mutant FLAG 3 -STAG3) and REC8-EGFP, or FLAG 3 -STAG3 (or mutant FLAG 3 -STAG3) and SMC1A-HA. Untransfected HEK293 cells were used as the negative control. The culture medium was supplemented with nocodazole for prometaphase arrest of the cells after $36 \mathrm{~h}$. Total protein was extracted after $12 \mathrm{~h}$ for Co-IP analysis. Wild-type STAG3 interacted with both REC8 and SMC1A, whereas there was no interaction between the three mutant STAG3 proteins with REC8 or SMC1A. WT indicates the plasmid encoding the wild type STAG3, Mut 1 indicates the plasmid encoding c.877_885del (p.293_295del) STAG3, Mut 2 indicates the plasmid encoding c.891_893dupTGA (p.297_298insAsp) STAG3, Mut 3 indicates the plasmid simultaneously encoding c.877_885del (p.293_295del) and c.891_893dupTGA (p.297_298insAsp) STAG3, and EV indicates the empty vector (without STAG3). 
p.297_298insAsp) of STAG3 were identified in a consanguineous Han Chinese family with POI. A fluorescence localization analysis was employed to evaluate the effects of the two variants and revealed that the mutant STAG3 proteins led to aberrant localization of REC8. Furthermore, interactions between the mutant STAG3 proteins and REC8 or SMC1A were absent, which was confirmed by Co-IP analysis. Therefore, both in-frame variants were shown to be deleterious and associated with POI in this family.

The STAG3 protein contains two domains: the STAG domain and the armadillo (ARM)-type domain. The ARM-type domain is located after the STAG domain, which is predicted to interact with nucleic acid or another protein (Caburet et al., 2014) (Figure 1E). At present, six STAG3 variants have been reported in five families with POI (Caburet et al., 2014; Stabej et al., 2016; Colombo et al., 2017; He et al., 2018a; Franca et al., 2019) (Table 1). All six variants are truncated variants that severely disrupt the STAG domain and/or ARM-type domain. Even c. $1573+5 \mathrm{G}>\mathrm{A}$ is a splice site variant, with RT-PCR revealing that the resulting mutant STAG3 protein is truncated (p.Leu490Thrfs ${ }^{\star}$ 10) (He et al., 2018a), leading to destruction of the ARM-type domain. In our study, both variants (p.293_295del and p.297_298insAsp) are in-frame variants, and neither localizes to the STAG or ARM-type domain; both variants are positioned between these two domains (Figure 1E). Therefore, the STAG and ARM-type domains are not disrupted, in theory. However, the p.293_295 and p.297_298 sites are highly conserved across species (Figure 1D), and nontruncating variants located between two domains have also been reported to impair protein function and lead to disease (Xia et al., 2015). Therefore, we postulate that the two variants are pathogenic, and that the region connecting the STAG and ARM-type domains is significant for STAG3 function.

To verify the pathogenicity of the two variants, we performed in vitro functional analyses to determine whether STAG3 protein function was impaired. STAG3 encodes a meiosisspecific subunit of cohesin, a multiprotein complex that plays an essential role in the proper pairing of chromosomes, sister chromatid cohesion, and chromosome segregation (Caburet et al., 2014; Wolf et al., 2018). Furthermore, STAG3 has been reported to interact with the cohesion subunits REC8 and SMC1A, and the entry of REC8 into nuclei requires STAG3 (Prieto et al., 2001; Wolf et al., 2018). The results of the fluorescence localization analysis in this study showed that none of the three mutant STAG3 proteins could assist REC8 in entering nuclei and that nearly all of the STAG3 mutants were restricted to the cytoplasm. In addition, there were no interactions between the mutant STAG3 proteins and REC8 or SMC1A observed in the Co-IP analysis. Therefore, we propose that the two in-frame variants of STAG3 lead to STAG3 dysfunction and are responsible for POI in the two study patients.

The proband's affected sister is sterile and has a small uterus and streak gonads, which is consistent with patients with POI caused by other STAG3 variants (Table 1). In contrast, the uterus and ovaries of the proband in our study were not visualized by ultrasonography (Table 1). The difference in phenotype between the proband and her affected sister might be explained by the proband's affected sister receiving HRT before coming to our hospital. Her uterus became larger after 3 months of HRT, which is consistent with patients with POI caused by variants in MCM8 and BRCA2 (Tenenbaumrakover et al., 2015; Weinbergshukron et al., 2018). Both MCM8 and BRCA2 have been shown to be associated with meiosis and female infertility (Moynahan et al., 2001; Rodriguezmari et al., 2011; Gambus and Blow, 2013), and STAG3 is a meiosis-specific gene involved in female infertility. Furthermore, MCM8 and BRCA2 have been associated with ovarian dysgenesis in humans (Moynahan et al., 2001; Rodriguezmari et al., 2011; Gambus and Blow, 2013). Female stag $^{-1-}$ mice also present with severe and very early ovarian dysgenesis (Caburet et al., 2014). Therefore, we suggest that STAG3 might also be related to ovarian dysgenesis in humans.

In summary, to the best of our knowledge, we are the first researchers to report in-frame variants of STAG3 that cause POI and to verify the role of in-frame STAG3 variants in POI pathogenicity through in vitro functional analyses of cell model. Our findings extend the mutational and phenotypic spectrums of STAG3 and have important implications for genetic counseling of patients with POI. However, to confirm the relationship between STAG3 and ovarian dysgenesis in humans, further study with a large sample size is needed.

\section{DATA AVAILABILITY STATEMENT}

The raw data supporting the conclusions of this manuscript will be made available by the authors, without undue reservation, to any qualified researcher.

\section{AUTHOR CONTRIBUTIONS}

JD and Y-QT designed the study. W-JX, W-BH, Y-XZ and L-LM performed the variant analysis of STAG3. W-JX and $\mathrm{W}-\mathrm{BH}$ carried out the evaluation of the pathogenicity of variations by in vitro cell model functional analyses. G-XL and GL worked on the clinical study. W-JX, W-BH, Y-QT and JD wrote the paper. All authors read and approved the final manuscript.

\section{FUNDING}

This work was supported by grants from the National Key Research \& Developmental Program of China (2018YFC1004900), the National Natural Science Foundation of China (81771645 and 81971447), the Hunan Provincial Natural Science Foundation of China (2019JJ51006), the Scientific Research Foundation of the Health Committee of Hunan Province (C2019193), the science and technology major project of the ministry of science and technology of Hunan Province, China (2017SK1030), and the Scientific Research Foundation of Reproductive and Genetic Hospital of CITIC-Xiangya (YNXM-201915, YNXM-201913, YNXM-201912, YNXM-201916). 


\section{ACKNOWLEDGMENTS}

We thank all of the donors who participated in this program and all of our colleagues at Reproductive and Genetic Hospital of CITIC-Xiangya.

\section{REFERENCES}

Bannatyne, P., Russell, P., and Shearman, R. P. (1990). Autoimmune oophoritis: a clinicopathologic assessment of 12 cases. Int J. Gynecol. Pathol. 9 (3), 191-207. doi: 10.1097/00004347-199007000-00001

Biscup, P. (2003). Risks and benefits of long-term hormone replacement therapy. Am. J. Health-syst. Pharm. 60 (14), 1419-1425. doi: 10.1093/ajhp/60.14.1419

Caburet, S., Arboleda, V. A., Llano, E., Overbeek, P. A., Barbero, J. L., Oka, K., et al. (2014). Mutant Cohesin in Premature Ovarian Failure. N. Engl. J. Med. 370 (10), 943-949. doi: 10.1056/NEJMoa1309635

Colombo, R., Pontoglio, A., and Bini, M. (2017). A STAG3 missense mutation in two sisters with primary ovarian insufficiency. Eur. J. Obstet. Gynecol. Reprod. Biol. 216, 269-271. doi: 10.1016/j.ejogrb.2017.08.005

Franca, M. M., Nishi, M. Y., Funari, M. F. A., Lerario, A. M., Baracat, E. C., Hayashida, S. A. Y., et al. (2019). Two rare loss-of-function variants in the STAG3 gene leading to primary ovarian insufficiency. Eur. J. Med. Genet. 62 (3), 186-189. doi: 10.1016/j.ejmg.2018.07.008

Gambus, A., and Blow, J. J. (2013). Mcm8 and Mcm9 form a dimeric complex in Xenopus laevis egg extract that is not essential for DNA replication initiation. Cell. Cycle. 12 (8), 1225-1232. doi: 10.4161/cc.24310

Garciacruz, R., Brieno, M. A., Roig, I., Grossmann, M., Velilla, E., Pujol, A., et al. (2010). Dynamics of cohesin proteins REC8, STAG3, SMC1 $\beta$ and SMC3 are consistent with a role in sister chromatid cohesion during meiosis in human oocytes. Hum. Reprod. 25 (9), 2316-2327. doi: 10.1093/humrep/deq180

Gowri, V., Al, S. M., Al-Farsi, F. A., Al-Busaidi, N. A., Dennison, D., Al, K. S., et al. (2015). Aetiological profile of women presenting with premature ovarian failure to a single tertiary care center in Oman. Post. Reprod. Health. 21 (2), 63-68. doi: 10.1177/2053369115587419

He, W., Banerjee, S., Meng, L., Du, J., Gong, F., Huang, H., et al. (2018a). Wholeexome sequencing identifies a homozygous donor splice-site mutation in STAG3 that causes primary ovarian insufficiency. Clin. Genet. 93 (2), 340-344. doi: $10.1111 /$ cge.13034

He, W., Tu, C., Liu, Q., Meng, L., Yuan, S., Luo, A., et al. (2018b). DMC1 mutation that causes human non-obstructive azoospermia and premature ovarian insufficiency identified by whole-exome sequencing. J. Med. Genet. 55 (3), 198-204. doi: 10.1136/jmedgenet-2017-104992

Houmard, B. S., Small, C., Yang, L., Naluaicecchini, T., Cheng, E., Hassold, T. J., et al. (2009). Global gene expression in the human fetal testis and ovary. Biol. Reprod. 81 (2), 438-443. doi: 10.1095/biolreprod.108.075747

Moynahan, M. E., Pierce, A. J., and Jasin, M. (2001). BRCA2 is required for homology-directed repair of chromosomal breaks. Mol. Cell 7 (2), 263-272. doi: 10.1016/S1097-2765(01)00174-5

Nogues, C., Fernandez, C., Rajmil, O., and Templado, C. (2009). Baseline expression profile of meiotic-specific genes in healthy fertile males. Fertil. Steril. 92 (2), 578-582. doi: 10.1016/j.fertnstert.2008.06.034

Prieto, I., Suja, J. A., Pezzi, N., Kremer, L., Martineza, C., Rufas, J. S., et al. (2001). Mammalian STAG3 is a cohesin specific to sister chromatid arms in meiosis I. Nat. Cell Biol. 3 (8), 761-766. doi: 10.1038/35087082

\section{SUPPLEMENTARY MATERIAL}

The Supplementary Material for this article can be found online at: https://www.frontiersin.org/articles/10.3389/fgene.2019.01016/ full\#supplementary-material

Richards, S., Aziz, N., Bale, S., Bick, D., Das, S., Gastier-Foster, J., et al. (2015) Standards and guidelines for the interpretation of sequence variants: a joint consensus recommendation of the American College of Medical Genetics and Genomics and the Association for Molecular Pathology. Genet. Med. 17 (5), 405-424. doi: 10.1038/gim.2015.30

Rodriguezmari, A., Wilson, C., Titus, T. A., Canestro, C., Bremiller, R., Yan, Y., et al. (2011). Roles of brca2 (fancd1) in oocyte nuclear architecture, gametogenesis, gonad tumors, and genome stability in zebrafish. PLoS Genet 7 (3), e1001357. doi: 10.1371/journal.pgen.1001357

Rossetti, R., Ferrari, I., Bonomi, M., and Persani, L. (2017). Genetics of primary ovarian insufficiency. Clin. Genet. 91 (2), 183-198. doi: 10.1111/cge.12921

Shelling, A. N. (2010). Premature ovarian failure. Reprod. 140 (5), 633-641. doi: 10.1530/REP-09-0567

Stabej, P. L. Q., Williams, H. J., James, C., Tekman, M., Stanescu, H., Kleta, R., et al. (2016). STAG3 truncating variant as the cause of primary ovarian insufficiency. Eur. J. Hum. Genet. 24 (1), 135-138. doi: 10.1038/ejhg.2015.107

Tenenbaumrakover, Y., Weinbergshukron, A., Renbaum, P., Lobel, O., Eideh, H., Gulsuner, S., et al. (2015). Minichromosome maintenance complex component 8 (MCM8) gene mutations result in primary gonadal failure. J. Med. Genet. 52 (6), 391-399. doi: 10.1136/jmedgenet-2014-102921

Tucker, E. J., Grover, S., Bachelot, A., Touraine, P., and Sinclair, A. H. (2016). Premature ovarian insufficiency: new perspectives on genetic cause and phenotypic spectrum. Endocr. Rev. 37 (6), 609-635. doi: 10.1210/er.2016-1047

Webber, L., Davies, M., Anderson, R. A., Bartlett, J., Braat, D., Cartwright, B., et al. (2016). ESHRE Guideline: management of women with premature ovarian insufficiency. Hum. Reprod. 31 (5), 926-937. doi: 10.1093/humrep/dew027

Weinbergshukron, A., Rachmiel, M., Renbaum, P., Gulsuner, S., Walsh, T., Lobel, O., et al. (2018). Essential role of brca2 in ovarian development and function. N. Engl. J. Med. 379 (11), 1042-1049. doi: 10.1056/NEJMoa1800024

Wolf, P. G., Ramos, A. C., Kenzel, J., Neumann, B., and Stemmann, O. (2018). Studying meiotic cohesin in somatic cells reveals that Rec8-containing cohesin requires Stag3 to function and is regulated by Wapl and sororin. J. Cell Sci. 131 (11), jcs212100. doi: $10.1242 /$ jcs.212100

Xia, H., Huang, X., Guo, Y., Hu, P., He, G., Deng, X., et al. (2015). Identification of a novel MYO15A mutation in a chinese family with autosomal recessive nonsyndromic hearing loss. PLoS One 10 (8), e0136306. doi: 10.1371/journal. pone. 0136306

Conflict of Interest: The authors declare that the research was conducted in the absence of any commercial or financial relationships that could be construed as a potential conflict of interest.

Copyright (C) 2019 Xiao, He, Zhang, Meng, Lu, Lin, Tan and Du. This is an open-access article distributed under the terms of the Creative Commons Attribution License (CC BY). The use, distribution or reproduction in other forums is permitted, provided the original author(s) and the copyright owner(s) are credited and that the original publication in this journal is cited, in accordance with accepted academic practice. No use, distribution or reproduction is permitted which does not comply with these terms. 